\title{
Ratio of In-Sphere Volume to Polyhedron Volume of the Great Pyramid Compared to Selected Convex Polyhedral Solids
}

\author{
Hans Hermann Otto \\ Materials Science and Crystallography, Clausthal University of Technology, Clausthal-Zellerfeld, Germany \\ Email: hhermann.otto@web.de
}

How to cite this paper: Otto, H.H. (2021) Ratio of In-Sphere Volume to Polyhedron Volume of the Great Pyramid Compared to Selected Convex Polyhedral Solids. Journal of Applied Mathematics and Physics, 9, 41-56.

https://doi.org/10.4236/jamp.2021.91005

Received: December 4, 2020

Accepted: January 4, 2021

Published: January 7, 2021

Copyright $\odot 2021$ by author(s) and Scientific Research Publishing Inc. This work is licensed under the Creative Commons Attribution International License (CC BY 4.0).

http://creativecommons.org/licenses/by/4.0/

\begin{abstract}
The architecture of the Great Pyramid at Giza is based on fascinating golden mean geometry. Recently the ratio of the in-sphere volume to the pyramid volume was calculated. One yields as result $R_{V}=\pi \cdot \varphi^{5}$, where $\varphi=\frac{\sqrt{5}-1}{2}$ is the golden mean. It is important that the number $\varphi^{5}$ is a fundamental constant of nature describing phase transition from microscopic to cosmic scale. In this contribution the relatively small volume ratio of the Great Pyramid was compared to that of selected convex polyhedral solids such as the Platonic solids respectively the face-rich truncated icosahedron (bucky ball) as one of Archimedes' solids leading to effective filling of the polyhedron by its insphere and therefore the highest volume ratio of the selected examples. The smallest ratio was found for the Great Pyramid. A regression analysis delivers the highly reliable volume ratio relation $R_{V}=1-\exp \left(-b \cdot \sqrt{n_{F}-3}\right)$, where $n_{F}$ represents the number of polyhedron faces and $b$ approximates the silver mean. For less-symmetrical solids with a unique axis (tetragonal pyramids) the in-sphere can be replaced by a biaxial ellipsoid of maximum volume to adjust the $R_{V}$ relation more reliably.
\end{abstract}

\section{Keywords}

Polyhedron, Great Pyramid, Platonic Solids, Volume-Area Ratio, In-Sphere and In-Ellipsoid, Polyhedral Void Space, Golden and Silver Mean

\section{Introduction}

Recently the golden mean architecture of the Great Pyramid at Giza was investigated with a surprising result [1]. Performing the volume ratio of the in-sphere 
of the pyramid and the pyramid itself, one yields as result $\pi \cdot \varphi^{5}$, where $\varphi=\frac{\sqrt{5}-1}{2}=0.6180339887$ is the golden mean. The same result can be obtained when calculating the respective areas ratio. This connection is valid for all regular convex polyhedral solids. Unfortunately, in the appendix of reference [1] the area ratio was carelessly calculated violating this proved fact. However, this flaw was already corrected in the prepublication [2].

In this contribution the in-sphere to polyhedron volume ratios for selected convex solids such as Platonic solids [3] respectively Archimedean ones [4] were compared to the result of the Great Pyramid. Face-rich regular solids adapt the in-sphere better to the polyhedron surface and exhibit a high volume ratio (see Figure 1). A simple relation is given to connect the volume ratio to the number of faces. It can be applied for the determination of the void space of polyhedral assemblies. For solids with reduced symmetry such as pyramids the in-sphere has to be replaced by a biaxial ellipsoid with maximum volume to adjust the relationship between volume ratio and number of faces more reliably.

\section{Methods}

Known elementary techniques of Euclidian coordinate geometry in $\mathbb{R}^{3}$ were used to determine area, volume, in-sphere volume and the in-sphere to polyhedron volume ratio for selected convex polyhedra, using the universal number of the golden mean when dealing with polyhedra of icosahedral symmetry. In contrast to irregular solids [5] [6] not being considered in this contribution, the analytic approach for regular solids is trivial. For solids with lower than cubic symmetry such as tetragonal pyramids, the in-sphere has to be replaced by an in-ellipsoid of maximum volume determining the zero of the first derivative of volume to half-diameter splitting. Then half-diameters were fixed by solving the ellipsoid equation using coordinates of two independent contact faces of the actual convex solid. Finally, a regression analysis delivered a reliable relation between volume ratio and number of faces.

\section{In-Sphere Approach}

For a convex polyhedron the radius of its in-sphere $r_{i}$ is related to the ratio between its volume $V_{p}$ and its surface area $A_{p}$ by

$$
r_{i}=3 \frac{V_{p}}{A_{p}}
$$

It could not be found out who actually first derived this relationship. However, in the Appendix an explanation for the more general application of the relation is given as the weighted mean "in-sphere" half-distance when the polyhedral void can't be adapted by a simple sphere, exemplified by the case of the truncated octahedron.

One can derive for the volume ratio using relation (1) 


$$
\frac{V_{s p h}}{V_{p}}=36 \pi \frac{V_{p}^{2}}{A_{p}^{3}}
$$

and further for the surface area ratio

$$
\frac{A_{s p h}}{A_{p}}=36 \pi \frac{V_{p}^{2}}{A_{p}^{3}}
$$

This proves that volume ratio and surface area ratio are equal

$$
\frac{V_{s p h}}{V_{p}}=\frac{A_{s p h}}{A_{p}}
$$

In the following the mentioned ratios were calculated for selected convex solids. The polyhedron notation is given by the symbol $\left[p_{i}^{F_{i}}\right]$, where $p$ is the polygon multiplicity and $F$ is the number of faces. The number $V$ of vertices can be derived by means of the following equation [7]

$$
V=\frac{1}{2} \sum p_{i} F_{i}-\sum F_{i}+2
$$

using the Euler relation for convex polyhedra [8]

$$
F+V-E=2
$$

where $E=\frac{1}{2} \sum p_{i} F_{i}$ means the number of edges. Don't confuse $V$ with symbols of Table 1 .

A list of notations used in the following was summarized in Table 1.

We begin with the truncated icosahedron, a convex isogonal non-prismatic solid of symmetry group $I_{h}$ with 32 faces, known from the soccer ball structure of $\mathrm{C}_{60}$, where $60 \mathrm{C}$ atoms are placed at the 60 vertices. The symmetry group tells us that the golden mean must be involved in the formulas for the polyhedron volume respectively area.

Table 1. List of used symbols and numbers.

\begin{tabular}{cc}
\hline Term & Symbol \\
\hline Polyhedral volume & $V_{p}$ \\
In-sphere volume & $V_{s p h}$ \\
In-ellipsoid volume & $V_{e l}$ \\
Polyhedral area & $A_{p}$ \\
In-sphere area & $A_{s p h}$ \\
In-sphere radius & $r_{i}$ \\
In-ellipsoid radii & $r_{x}, r_{z}$ \\
Polyhedron edge & $a$ \\
Polyhedron height & $h$ \\
Golden mean & $\varphi$ \\
Big phi & $\phi=\varphi+1$
\end{tabular}


Truncated icosahedron (soccer ball) $\left[5^{12} 6^{20}\right]$ :

$$
\begin{gathered}
V_{p}=\left(21 \varphi^{-2}+\frac{\varphi}{2}\right) a^{3}=55.2877308 \cdot a^{3} \\
A_{p}=\left(20 \cdot \frac{3}{2} \sqrt{3}+12 \frac{5}{4 \cdot \sqrt{\varphi^{3} \sqrt{5}}}\right) a^{2}=72.607253 \cdot a^{2} \\
r_{i}=3 \frac{V_{p}}{A_{p}}=\frac{21 \varphi^{-2}+\frac{\varphi}{2}}{5\left(2 \sqrt{3}+\left(\varphi^{3} \sqrt{5}\right)^{-\frac{1}{2}}\right)} a=2.284388754 \cdot a \approx \sqrt{2} \varphi^{-1} a \\
V_{s p h}=\pi \cdot \frac{6^{2}}{5^{3}}\left(\begin{array}{c}
\left.7 \varphi^{-2}+\frac{\varphi}{6}\right)^{3} \\
\left.2 \sqrt{3}+\left(\varphi^{3} \sqrt{5}\right)^{-\frac{1}{2}}\right)^{3}=\pi \cdot 15.89456977 \cdot a^{3} \approx \pi \cdot\left(\frac{4}{3}\right)^{5 / 4} \cdot \varphi^{-5} a^{3} \\
\frac{V_{s p h}}{V_{p}}=\pi \cdot 36 \frac{V_{p}^{2}}{A_{p}^{3}}=\pi \cdot 0.28748819=0.903170795 \\
A_{s p h}=4 \pi r_{i}^{3} a^{2}=\pi \cdot 36\left(\frac{V_{p}}{A_{p}}\right)^{2} a^{2} \\
\frac{A_{s p h}}{A_{p}}=\pi \cdot 36 \frac{V_{p}^{2}}{A_{p}^{3}}
\end{array}\right.
\end{gathered}
$$

Triacontahedron $\left[4^{30}\right]$ (Kepler zonohedron $\mathrm{K}_{30}$ see Appendix):

$$
\begin{gathered}
V_{p}=4 \sqrt{5+2 \sqrt{5}} a^{3} \\
A_{p}=12 \sqrt{5} a^{2}=26.8328157 \cdot a^{2} \\
r_{i}=\sqrt{\frac{5+2 \sqrt{5}}{5}} a=1.37638192 \cdot a \\
\frac{V_{s p h}}{V_{p}}=\frac{4}{3} \pi r_{i}^{3}=\pi \cdot \frac{\varphi^{-3}}{15}=0.8872000 \\
A_{s p h}=4 \pi\left(1+\frac{2}{\sqrt{5}}\right) a^{2}=23.806074 \cdot a^{2} \\
\frac{A_{s p h}}{A_{p}}=4 \pi r_{i}^{2}=\pi \cdot \frac{\varphi^{-3}}{15}=0.8872000
\end{gathered}
$$

Hexakishexahedron $\left[3^{24}\right]$ :

$$
\begin{gathered}
V_{p}=\frac{3}{2} a^{3} \\
r_{i}=\frac{3}{2 \sqrt{5}} a \\
V_{s p h}=\pi \cdot \frac{9}{10 \sqrt{5}} a^{3}=1.2644666 \cdot a^{3}
\end{gathered}
$$




$$
\begin{gathered}
\frac{V_{s p h}}{V_{p}}=\pi \cdot \frac{3}{5 \sqrt{5}}=0.842977767 \\
A_{p}=3 \sqrt{5} a^{2} \\
A_{s p h}=\pi \cdot \frac{9}{5} a^{2}=5.65486677 \cdot a^{2} \\
\frac{A_{s p h}}{A_{p}}=\pi \cdot \frac{3}{5 \sqrt{5}}=\pi \cdot \frac{3}{\varphi^{-5}+\varphi^{5}}=0.842977767
\end{gathered}
$$

\section{Icosahedron $\left[3^{20}\right]$ :}

$$
\begin{aligned}
& V_{p}=\frac{5}{6} \varphi^{-2} a^{3} \\
& r_{i}=\frac{\varphi^{-2}}{2 \sqrt{3}} a \\
& V_{s p h}=\pi \cdot \frac{\varphi^{-6}}{18 \sqrt{3}} a^{3} \\
& \frac{V_{s p h}}{V_{p}}=\pi \cdot \frac{\varphi^{-4}}{15 \sqrt{3}}=\pi \cdot 0.263814507=0.8287977 \\
& A_{p}= 5 \cdot \sqrt{3} a^{2} \\
& A_{s p h}=\pi \frac{\varphi^{-4}}{3} a^{2} \\
& \frac{A_{s p h}}{A_{p}}=\pi \cdot \frac{\varphi^{-4}}{15 \sqrt{3}}
\end{aligned}
$$

Truncated Octahedron $\left[4^{6} 6^{8}\right]$ ( $\alpha$-cage of the sodalite structure): (Combination of the octahedron with its dual polyhedron)

Like the cube this polyhedron is space-filling. However, the distances from the center to the different polygon faces are also different, and we apply a weighted in-sphere radius as given by equation one. The reason for this approximation is explained in the Appendix.

$$
\begin{gathered}
V_{p}=8 \sqrt{2} a^{3}=11.3137085 \cdot a^{3} \\
r_{i}=\frac{4 \sqrt{2}}{1+2 \sqrt{3}} a=1.2671876 \cdot a \\
V_{s p h}=\frac{4}{3} \pi \cdot\left(\frac{4 \sqrt{2}}{1+2 \sqrt{3}}\right)^{3} \cdot a^{3}=8.523370381 \cdot a^{3} \\
\frac{V_{s p h}}{V_{p}}=0.2398040 \cdot \pi=0.753366624 \\
A_{p}=6(1+2 \sqrt{3}) a^{2}=26.7846097 \cdot a^{2} \\
A_{s p h}=4 \pi\left(\frac{4 \sqrt{2}}{1+2 \sqrt{3}}\right)^{2} \cdot a^{2}=6.42305774 \cdot \pi \cdot a^{2}
\end{gathered}
$$




$$
\frac{A_{s p h}}{A_{p}}=\frac{\pi}{3}\left(\frac{4}{1+2 \sqrt{3}}\right)^{3}=0.2398040 \cdot \pi=0.753366624
$$

Regular Pentagonal Dodecahedron [5 $\left.5^{12}\right]$ :

$$
\begin{gathered}
V_{p}=\frac{\sqrt{5}}{2 \varphi^{4}} a^{3}=\frac{5}{2 \varphi^{3}\left(1+\varphi^{2}\right)} a^{3}=7.6631188998 \cdots a^{3} \\
r_{i}=\frac{1}{2 \sqrt{\sqrt{5} \varphi^{5}}} a=\frac{1}{2 \varphi^{2} \cdot \sqrt{1+\varphi^{2}}} a=1.113516364 \cdots a \\
V_{s p h}=\frac{4}{3} \pi r_{i}^{3}=\pi \cdot 1.840893008 \cdots a^{3} \\
\frac{V_{s p h}}{V_{p}}=0.240227643 \cdot \pi=0.754697398 \\
A_{p} \cdot \sqrt{5(5+2 \sqrt{5})} \cdot a^{2}=\frac{15}{\varphi \sqrt{1+\varphi^{2}}} a^{2}=20.64572881 \cdots a^{2} \\
A_{s p h}=4 \pi r_{i}^{2}=\pi \frac{1}{\sqrt{5} \varphi^{5}} a^{2}=\pi \cdot 4.9596747 \cdots a^{2} \\
\frac{A_{s p h}}{A_{p}}=0.240227643 \cdot \pi=0.754697398
\end{gathered}
$$

Rhombic Dodecahedron $\left[4^{12}\right]$ :

$$
\begin{gathered}
V_{p}=\frac{16}{3 \sqrt{3}} a^{3} \\
r_{i}=\frac{\sqrt{2}}{\sqrt{3}} a \\
V_{s p h}=\frac{4}{3} \pi\left(\frac{\sqrt{2}}{\sqrt{3}}\right)^{3} a^{3}=\pi \cdot \frac{8 \sqrt{6}}{27} a^{3} \\
\frac{V_{s p h}}{V_{p}=\pi \cdot \frac{\sqrt{2}}{6}=\pi \cdot 0.23570226=0.74048048} \\
A_{p}=8 \sqrt{2} a^{2} \\
\frac{A_{s p h}}{A_{p}}=\pi \cdot \frac{2}{3 \sqrt{2}}=\pi \cdot 0.23570226
\end{gathered}
$$

Octahedron $\left[3^{8}\right]$ :

$$
\begin{gathered}
V_{p}=\frac{\sqrt{2}}{3} a^{3} \\
r_{i}=\frac{a}{\sqrt{6}}
\end{gathered}
$$




$$
\begin{gathered}
V_{s p h}=\frac{4}{3} \pi\left(\frac{a}{\sqrt{6}}\right)^{3} \\
\frac{V_{s p h}}{V_{p}}=\pi \frac{1}{3 \sqrt{3}}=\pi \cdot 0.192450089=0.604599788 \\
A_{p}=2 \sqrt{3} a^{2} \\
A_{s p h}=4 \pi\left(\frac{a}{\sqrt{6}}\right)^{2}=\frac{2}{3} \pi \\
\frac{A_{s p h}}{A_{p}}=\pi \frac{1}{3 \sqrt{3}}
\end{gathered}
$$

Hexahedron (Cube) $\left[4^{6}\right]$ :

$$
\begin{gathered}
V_{p}=a^{3} \\
V_{s p h}=\frac{\pi}{6} a^{3} \\
\frac{V_{s p h}}{V_{p}}=\frac{\pi}{6}=0.523598775 \\
A_{p}=6 a^{2} \\
A_{s p h}=4 \pi\left(\frac{a}{2}\right)^{2}=\pi a^{2} \\
\frac{A_{s p}}{A_{p}}=\frac{\pi}{6}
\end{gathered}
$$

Tetrahedron $\left[3^{4}\right]$ (self-dual polyhedron):

$$
\begin{gathered}
V_{p}=\frac{\sqrt{2}}{12} a^{3} \\
r_{i}=\frac{1}{2 \sqrt{6}} a \\
V_{s p h}=\frac{4}{3} \pi r_{i}^{3}=\frac{4}{3} \pi\left(\frac{1}{2 \sqrt{6}}\right)^{3} a^{3} \\
\frac{V_{s p h}}{V_{p}}=\frac{\pi}{\sqrt{3} \cdot 6}=\pi \cdot 0.096225044=0.30229989 \\
A_{p}=\sqrt{3} a^{2} \\
\left.\frac{A_{s p h}=}{\frac{A_{s p h}}{A_{p}}}=\frac{\pi}{6 \sqrt{6}}\right)^{2} a^{2}=\frac{\pi}{6} a^{2}
\end{gathered}
$$

Equilateral Quadratic Pyramid (Half-Octahedron) $\left[3^{4} 4^{1}\right]$ :

$$
V_{p}=\frac{a^{3}}{\sqrt{2} \cdot 3}
$$




$$
\begin{gathered}
r_{i}=\frac{a}{\sqrt{2}(1+\sqrt{3})} \\
V_{s p h}=\frac{4}{3} \pi r_{i}^{3}=\frac{\sqrt{2} \pi a^{3}}{3(1+\sqrt{3})^{3}} \\
\frac{V_{s p h}}{V_{p}}=\pi \cdot \frac{2}{(1+\sqrt{3})^{3}}=\pi \cdot 0.0980762=0.308115505 \\
A_{p}=(1+\sqrt{3}) a^{2} \\
\frac{A_{s p}}{A_{s p h}}=4 \pi \frac{a^{2}}{2(1+\sqrt{3})^{2}} \\
A_{p}=\frac{2 \pi}{(1+\sqrt{3})^{3}}=\pi \cdot 0.0980762=0.308115505
\end{gathered}
$$

\section{Great Pyramid $\left[3^{4} 4^{1}\right]$ :}

In case of the Great Pyramid we use exceptionally for the basis length the value $a=2$ in accordance with a recently published contribution [1].

$$
\begin{gathered}
V_{p}=\frac{1}{3} a^{2} h=\frac{4}{3} \varphi^{-1 / 2} \\
r_{i}=\varphi^{3 / 2} \\
V_{s p h}=\frac{4}{3} \pi r^{3}=\frac{4}{3} \pi \varphi^{9 / 2} \\
\frac{V_{s p h}}{V_{p}=\pi \cdot \varphi^{5}=}=\pi \cdot 0.0901699=0.283277 \\
A_{p}=4 \varphi^{-1}+4=4 \varphi^{-2} \\
A_{s p h}=4 \pi r^{2}=4 \pi \varphi^{3} \\
\frac{A_{s p h}}{A_{p}}=\pi \cdot \varphi^{5}
\end{gathered}
$$

In Figure 1 the volume ratio $R_{V}$ is depicted in relation to the number of faces. However, because one needs a minimum of 4 faces to put up a solid, the number of faces was reduced by 3 . Therefore, a highly reliable least squares fit was performed using the relation

$$
R_{V}=1-\exp \left(-b \cdot \sqrt{n_{F}-3}\right)
$$

The parameter $b$ was found to be $0.418 \pm 0.011(2.6 \%)$. This value approximates $\sqrt{2}-1=0.414235 \cdots$ known as silver mean. The tetragonal pyramids (halfoctahedron and Great Pyramid) were not included in the fit, because they don't match the regression curve (see green marked solids in Figure 1) due to reduced symmetry. Polyhedral solids with icosahedral symmetry indicate a slightly enhanced volume ratio compared to these with cubic symmetry. However, diversification 


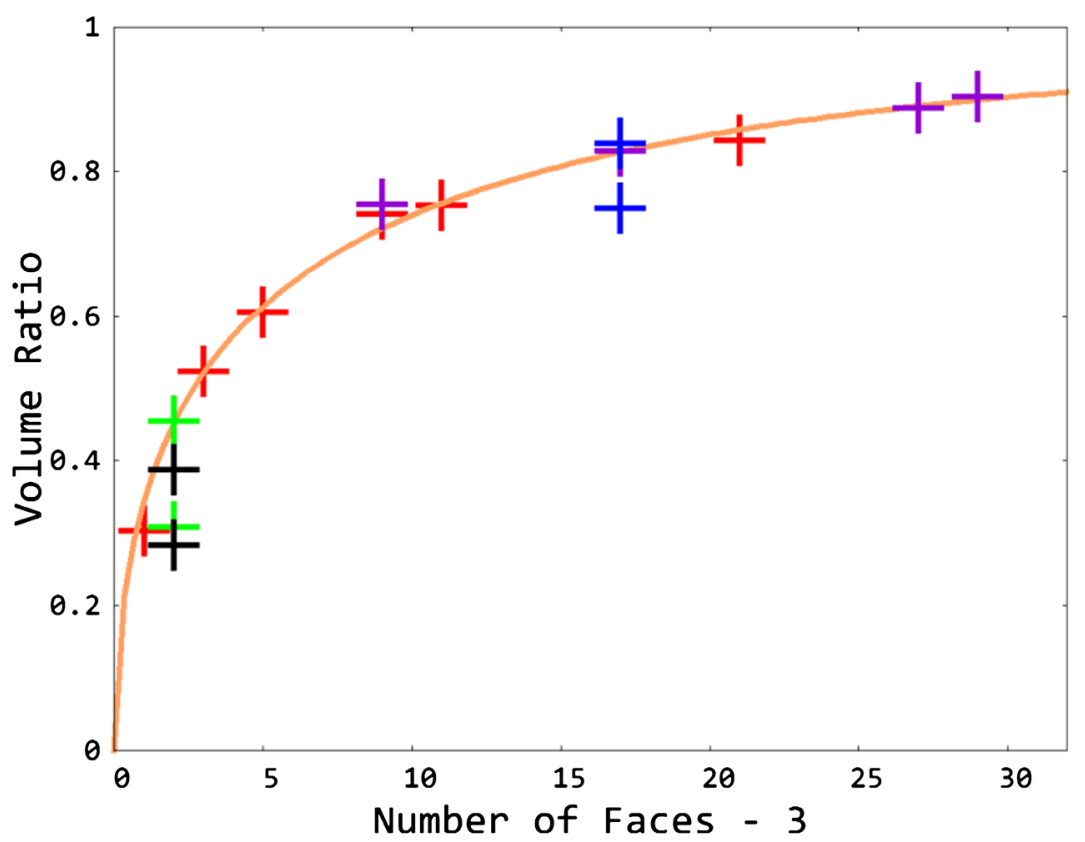

Figure 1. Volume ratio of selected solids related to their by 3 reduced face numbers. Red: solids with cubic symmetry, violet: solids with icosahedral symmetry. The green respectively black marked tetragonal pyramids (half-octahedron and Great Pyramid) as well as the blue data for the Bilinski B20 zonohedron were not included in the fit, because their smaller volume ratios behave somewhat differently.

of the plot does not give a profound improvement.

Applying Equation (89) to the case of a hexakisicosahedron with 120 triangular faces, one can predict for its volume ratio a value of $R_{V} \approx 0.99$. In practice, the polyhedral void space of polyhedral networks can approximately be determined by this formula.

Turning back to the deviation of the volume ratios for both quadratic pyramids from that of the fitted curve, denoted by $R_{V}^{\prime}$, one can determine the following approximations

$$
\begin{aligned}
& \text { Half-octahedron: } \frac{R_{V}^{\prime}}{\sqrt{2} R_{V}}=1.0229 \approx 1 \\
& \text { Great Pyramid: } \frac{5 R_{V}^{\prime}}{8 R_{V}}=0.9834 \approx 1
\end{aligned}
$$

Interestingly, the term 5/8 in equation (90) approximates the golden mean $\varphi$.

\section{Inscribed Ellipsoid Approach}

However, symmetry reduction from icosahedral respectively cubic symmetry to tetragonal one resulted in the degeneration of the in-sphere to a biaxial ellipsoid as approximated void space. The volume $V_{e}$ of a biaxial ellipsoid with $z$ being the rotation axis is given by

$$
V_{e}=\frac{4}{3} \pi r_{x}^{2} r_{z}
$$


where $r_{x}$ and $r_{z}$ are the corresponding half-axis.

\section{Equilateral Quadratic Pyramid}

In case of the half-octahedral pyramid the inscribed rotation ellipsoid of maximum volume has half-diameters of $r_{z}=r_{i}-\Delta_{\max }$ and $r_{x}=r_{i}+\Delta_{\max } \cdot \sqrt{2}$. Replacing these relations in Equation (92), then the first derivative $\frac{\mathrm{d} V}{\mathrm{~d} \Delta}=0$ delivers

$$
\Delta_{\max }=\frac{2-\sqrt{2} / 2}{3} r_{i}=0.430964 \cdot r_{i} .
$$

The volume ratio is represented in Figure 1 with the top green cross and was calculated to be

$$
V_{e} / V_{p}=\frac{V_{s p h}}{V_{p}}\left(\frac{4}{27}\left(5+\frac{7}{\sqrt{2}}\right)\right)=0.45417 .
$$

This value fits relation (89) reasonable well. The numerical eccentricity of the maximum biaxial in-ellipsoid with $k=\frac{r_{z}}{r_{x}}$ yielded $\epsilon=\sqrt{1-k^{2}}=0.93539$.

\section{Great Pyramid}

Turning to the comparable results for the Great Pyramid, one has to work with ellipsoid half-diameters $r_{z}=r_{i}-\Delta_{\max }$ and $r_{x}=r_{i}+\Delta_{\max } / \sqrt{\varphi}$. Now $\Delta_{\max }$ is calculated to be

$$
\Delta_{\max }=\frac{2-\sqrt{\varphi}}{3} r_{i}=0.4046162 \cdot r_{i}
$$

giving for the half diameters

$$
r_{x}=\frac{2}{3} \varphi(1+\sqrt{\varphi})=0.735934838, r_{z}=\frac{1}{3} \varphi^{\frac{3}{2}}(1+\sqrt{\varphi})=0.28927809
$$

and for its ratio $k$ respectively eccentricity $\varepsilon$

$$
k=\frac{r_{z}}{r_{x}}=\frac{\sqrt{\varphi}}{2}=0.393075688, \varepsilon=\sqrt{1-k^{2}}=\frac{\sqrt{4-\varphi}}{2}=0.919506
$$

Then the volume $V_{e}$ simply results in

$$
V_{e}=\left(\frac{2}{3}\right)^{4} \varphi^{\frac{7}{2}}(1+\sqrt{\varphi})^{3} \cdot \pi=0.6562705
$$

Using relation (82) the ratio $\frac{V_{e}}{V_{p}}$ (represented in Figure 1 with the top black cross) numerically gives

$$
\frac{V_{e}}{V_{p}}=\frac{4}{27} \varphi^{4}(1+\sqrt{\varphi})^{3} \cdot \pi=0.386946
$$

This Great Pyramid volume ratio based on its maximum in-ellipsoid is still not quite well adapted to curve (89) in Figure 1. A comparison between in-sphere and in-ellipsoid of the Great Pyramid was illustrated in Figure 2.

Finally one should note that if the sphere degenerates to an ellipsoid, Equation 

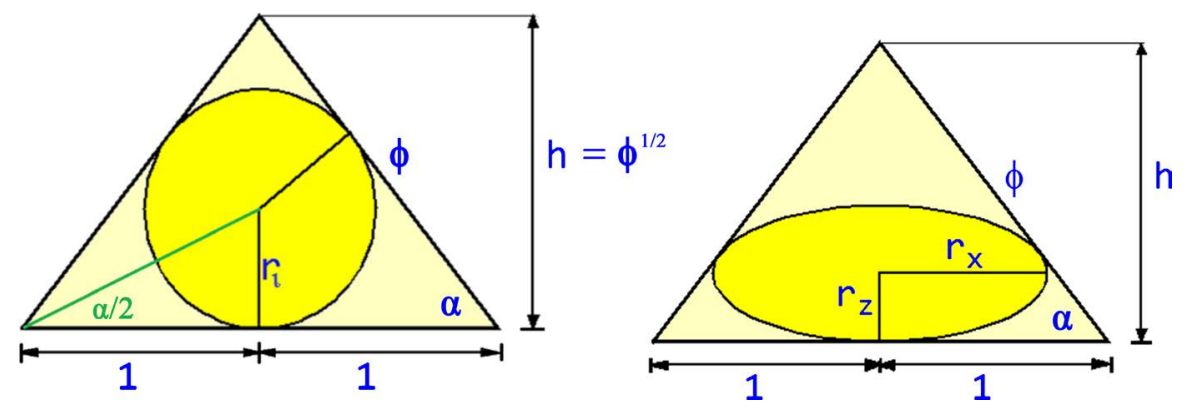

Figure 2. Cut through the middle of a Great Pyramid's face down the apex. In-sphere respectively in-ellipsoid projection yellow displayed. $\phi=\varphi^{-1}=1+\varphi$, $\alpha=\arccos (\varphi)=\arctan (\sqrt{\phi})=51.82729^{\circ}$.

(4) no longer holds, because

$$
\frac{A_{e}}{A_{p}}>\frac{V_{s p h}}{V_{p}}
$$

Again the case of the Great Pyramid should serve to demonstrate this. The surface $A$ of a biaxial ellipsoid with rotation axis $z$ and eccentricity $\epsilon=\sqrt{1-k^{2}}, k=\frac{r_{z}}{r_{x}}$ is given by

$$
A=2 \pi r_{x}^{2}\left(1+k^{2} \frac{\operatorname{atanh}(\varepsilon)}{\varepsilon}\right)
$$

The surface at maximum volume of the ellipsoid $A_{e}$ is then calculated as $A_{e}=2.093982$ and

$$
\text { the ratio } \frac{A_{e}}{A_{p}}=0.323538>\frac{A_{s p h}}{A_{p}}=\pi \cdot \varphi^{5}=0.283277
$$

\section{Bilinski B20 Zonohedron}

Finally, the in-ellipsoid approach was applied to the Bilinski B20 zonohedron as example for an oblate polyhedron with a unique five-fold axis, derived from the triacontahedron by removing a complete zone of 10 faces. The calculation of its in-ellipsoid half-diameters was given in the Appendix. Then one determines the ellipsoid volume according to Equation (92) as

$$
V_{e l}=5.165 a^{3}
$$

The volume of the $B 20$ polyhedron is one half of that of the triacontahedron

$$
V_{p}=2 \sqrt{5+2 \sqrt{5}} a^{3}=6.155367 a^{3}
$$

and the volume ratio, depicted by the top blue cross in Figure 1, yields

$$
R_{V}^{\prime}=\frac{V_{e l}}{V_{p}}=0.8391
$$

Contrary, the calculation of the volume ratio using an in-sphere radius defined by Equation (1) leads to a very low ratio, with $A_{p}=\frac{2}{3} A_{p}$ (triacontahedron) 


$$
\begin{gathered}
r_{i}=3 \frac{V_{p}}{A_{p}}=\frac{3}{4} r_{i}(\text { triacontahedron }) a=1.032286 a \\
V_{s p h}=\frac{4}{3} \pi r_{i}^{3} a^{3}=\frac{9 \pi}{16}\left(1+\frac{2}{\sqrt{5}}\right)^{3 / 2}=4.6077539 a^{3} \\
R_{V}=\frac{V_{s p h}}{V_{p}}=0.748575
\end{gathered}
$$

\section{Intended Application}

Information about the in-sphere of polyhedral solids as interstitial void space in networks of alumo-silicates (zeolites), metallic glasses, amorphous alloys or liquids are important to understand their packing density and related properties such as phase transitions [9] [10]. The maximum in-ellipsoid may be a challenging approach for new practical utilization. Especially a micro-version of the Great Pyramid may serve as proposal for an asymmetric 'confinement chamber' for nuclear fuel such as deuterium, provided you believe in the feasibility of cold nuclear fusion [11] [12]. In this way we walk in the footsteps of the great inventor Nicola Tesla with his pyramid vision of harvesting energy.

\section{Duality between Volume and Surface}

The relationship between volume and surface goes far beyond a purely geometric understanding. The duality between volume and surrounding surface respectively between any compact entity and assigned surface in general as well as the duality between a moving particle respectively body and the accompanying wave or reciprocity between matter and dark matter is the very spice of life. It has been impressively formulated by the words of Nobel laureate Wolfgang Pau1i: "God made the bulk; surfaces were invented by the devil" (quoted from [19]). These facts were once more proven by the beautiful new information relativity theory of Suleiman [20] [21].

\section{Conclusions}

This contribution was inspired by a flaw in a recently published own paper. It compares the ratio of in-sphere volume to polyhedral volume of selected convex solids, which is always equal to the corresponding ratio of in-sphere surface and polyhedral surface. The volume ratio of the Great Pyramid is the smallest among the selected solids and also smaller as the similar half-octahedral pyramid as well as the tetrahedron. Convex solids with icosahedral symmetry indicate a slightly higher volume ratio than solids with cubic symmetry respectively tetragonal symmetry. However, replacing the in-sphere by the maximum inscribed biaxial ellipsoid one can adjust the volume ratio more reliably to a proposed relationship that connects the volume ratio with the number of faces. The in-sphere volume ratio of the Great Pyramid was recently shown to be 
$\pi \cdot \varphi^{5}$, where $\varphi$ is the golden mean. Assigned as fundamental number of nature, the fifth power of the golden mean, on the other hand, is fascinating through its connection to phase transition from microscopic to cosmic scale and may become important in connection with the $D N A$ genetic code.

This number is also indirectly found in the expressions for the in-sphere surface of the regular pentagonal dodecahedron, not really expected in the term for the volume ratio of the hexakishexahedron, and as an approximation in the insphere volume relation for the soccer ball respectively the triacontahedron. Besides a solely geometrical interest, the theory of random packing respectively the determination of polyhedral void space as in-sphere volume respectively in-ellipsoid volume is important for practical applications.

\section{Acknowledgements}

I am indebted to P. P. Klein from the TU Clausthal, who made me known to a flaw in the Appendix of my recent work [1] and kindly helped improve this work. He contributed interesting relations to a similar theme [6]. The author acknowledged also the careful reading of the first draft by Leila Marek-Crnjac, Technical School Center of Maribor.

\section{Conflicts of Interest}

The author declares no conflicts of interest regarding the publication of this paper.

\section{References}

[1] Otto, H.H. (2020) Magic Numbers of the Great Pyramid: A Surprising Result. Journal of Applied Mathematics and Physics, 8, 2063-2071.

https://doi.org/10.4236/jamp.2020.810154

[2] Otto, H.H. (2020) Magic Numbers of the Great Pyramid: A Surprising Result. Researchgate.net.

[3] Archer-Hind, R.D. (1888) The Timaeus of Plato. MacMillan and Co., New York.

[4] Field, J.V. (1997) Rediscovering the Archimedean Polyhedra: Pierro della Francesca, Luca Pacioli, Leonardo da Vinci, Albrecht Dürer, Daniele Barbaro, and Johannes Kepler. Archive for History of Exact Sciences, 50, 241-289.

[5] Suhu, K.K. and Lahiri, A.K. (2004) Finding the Insphere of a Convex Polyhedron: An Analytical Approach. Philosophical Magazine, 84, 1185-1196. https://doi.org/10.1080/14786430310001647159

[6] Klein, P.P. (2020) The Insphere of a Tetrahedron. Applied Mathematics, 11, 601-612. https://doi.org/10.4236/am.2020.117041

[7] Otto, H.H. (2015) Modeling of a Cubic Antiferromagnetic Cuprate Super-Cage. World Journal of Condensed Matter Physics, 5, 160-178. https://doi.org/10.4236/wjcmp.2015.53018

[8] Euler, L. (1752) Elemanta Doctrine Solidorum. Novi commentarii academiae scientiarum imperialis petropolitanae, 4, 109-160.

[9] Bernal, J.D. (1964) The Bakterian Lecture, 1962. The Structure of Liquids. Proceedings of the Royal Society A, 280, 299-322. https://doi.org/10.1098/rspa.1964.0147 
[10] Finnay, J.L. (1977) Modelling the Structures of Amorphous Metals and Alloys. Nature, 266, 309-314. https://doi.org/10.1038/266309a0

[11] Garai, J. (2019) Physical Model for Lattice Assisted Nuclear Reactions. Conference Paper, 1-28.

[12] Otto, H.H. (2020) Nuclear Fusion Research and Development Need New Relativistic Mass and Energy Corrections Given by the Information Relativity Theory.

[13] Otto, H.H. and Ellner, M. (1987) Röntgenbeugungsuntersuchungen an quasikristallinem $\mathrm{All}_{4} \mathrm{Mn}$ (X-Ray Diffraction Analysis of Quasi-Crystalline I- $\mathrm{Al}_{4} \mathrm{Mn}$ ). Zeitschrift für Kristalligraphie, 178, 178-179.

[14] Otto, H.H. (2020) A Varied Quartic Polynomial Modeling the DNA Genetic Code.

[15] Otto, H.H. (2017) Should We Pay More Attention to the Relationship between the Golden Mean and the Archimedes' Constant? Nonlinear Science Letters A, 8, 410412.

[16] Bilinski, S. (1960) Über das Rhombenikosaeder. Glasnik Matematicko-Fizicki i Astronomski, 15, 251-263.

[17] Coxeter, H.M.S. (1975) Regular Polytopes. Second Edition, Dover Publications Inc., Mineola.

[18] Gray, R.W. (2007) Polyhedra Coordinates. In: Gray, R.W., Ed., Encyclopedia Polyhedra: Rhombic Triacontahedron, Internet Edition, 1-11.

[19] El Naschie, M.S., Olsen, S., Helal, M.A., Marec-Crnjac and Nada, S. (2018) On the Missing Link between Cosmology and Biology. International Journal of Innovation in Science and Mathematics, 6, 11-13.

[20] Suleiman, R. (2019) Relativizing Newton. Nova Scientific Publisher, New York, 1-207.

[21] Otto, H.H. (2020) Reciprocity as an Ever-Present Dual Property of Everything. Journal of Modern Physics, 11, 98-121. https://doi.org/10.4236/jmp.2020.111007 


\section{Appendix}

\section{Truncated Octahedron:}

The distances $r_{i, f}$ from the center to the different faces $f$ (square and hexagon) are calculated to be $r_{i, 4}=\sqrt{2} \cdot a$ and $r_{i, 6}=\frac{1}{2} \sqrt{6} \cdot a$. Now we use a weighted in-sphere radius, where the face areas are thought to be a measure of the radius of curvature. Then we get the weighted radius as

$$
r_{i}=\frac{\sqrt{2}+2 \sqrt{3} \cdot \frac{1}{2} \sqrt{6}}{1+2 \sqrt{3}} a=\frac{4 \sqrt{2}}{1+2 \sqrt{3}} a=1.267187608 \cdot a
$$

This weighted radius is identical to that used in Equation (35) derived by application of the relation $r_{i}=3 \frac{V_{p}}{A_{p}}$ underlining the universal importance of this relation. The volume ratio of the truncated octahedron is well represented by Equation (89) using this approach.

\section{Triacontahedron (Figure A1):}

It belongs to the icosahedral group $I_{h}$ and is a regular polyhedron consisting of 30 equilateral rhobus faces (golden rombi), 32 vertices and 60 edges. It was first discovered by Johannes Kepler (1571-1630). Many years ago I had chosen this solid serving as a structural model forthe first building sphere of quasi-crystals belonging to the Al-Mn alloy system, where neighboring polyhedra interpenetrate [13].

Interestingly, the volume ratio of the triacontahedron can by approximated by [14] [15]

$$
\frac{V_{s p h}}{V_{p}}=\frac{4}{3} \pi r_{i}^{3}=\pi \cdot \frac{\varphi^{-3}}{15}=0.8872000 \approx \frac{2}{25 \cdot \varphi^{5}}=0.88721359
$$

In this way, one is again faced with the fifth power of the golden mean.

\section{Bilinski Zonohedron}

If one remove a complete zone from the rhombic triacontahedron, for instance the middle zone parallel to the $c$ axis in Figure A1, then an oblate solid with 20 faces results named Bilinski's $B 20$ zonohedron [16], a solid that already

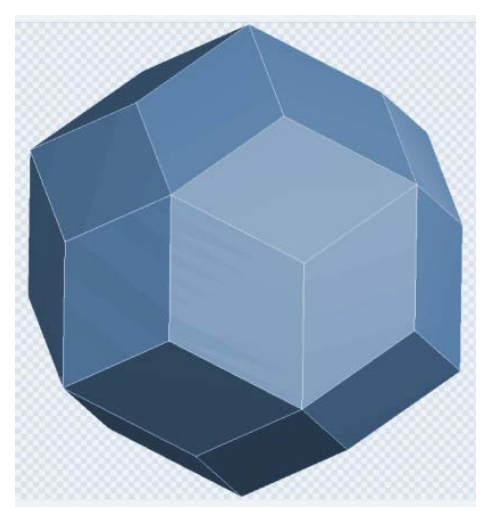

Figure A1. Rhombic Triacontahedron (Keplerhedron, notation K30). 
has figured out by Cowley in 1752 [17]. Due to symmetry reduction a biaxial ellipsoid instead of a sphere was fitted into the solid to approximate the volume ratio given by Equation (89).

Using two independent Cartesian face coordinates of the B20 zonohedron, choosing faces of different steepness, on can solve the ellipsoid equation to get the two half diameters $r_{x}$ and $r_{z}$ of the biaxial ellipsoid as

$$
\begin{gathered}
r_{x}=\sqrt{\frac{z_{2}^{2}\left(x_{1}^{2}+y_{1}^{2}\right)-z_{1}^{2}\left(x_{2}^{2}+y_{2}^{2}\right)}{z_{2}^{2}-z_{1}^{2}}} \\
r_{z}=\frac{r_{x} z_{1}}{\sqrt{r_{x}^{2}-x_{1}^{2}-y_{1}^{2}}}
\end{gathered}
$$

Coordinates of the triacontahedron given by Gray [18] were used, rotated that a fivefold axis is parallel to the $c$ axis and normed to an edge length of unity. The middle zone with zone axis parallel to $c$ is then cut out to deliver coordinates of the $B 20$ zonohedron. One obtains the following face coordinates using big $\phi=1+\varphi=1.6180339887 \cdots$

$$
\begin{gathered}
x_{1}=\frac{\phi}{\sqrt{5}} a, y_{1}=0, z_{1}=\frac{3}{2 \sqrt{5}} a \\
x_{2}=\frac{1}{\sqrt{5}}\left(\phi+\frac{1}{2}\right) a, y_{2}=\frac{1}{2} \sqrt{1+\frac{2}{\sqrt{5}}} a, z_{2}=\frac{1}{2 \sqrt{5}} a
\end{gathered}
$$

With these coordinates one yields the $B 20$ in-ellipsoid half-diameters

$$
r_{x}=1.21520448 a \text { and } r_{z}=0.834993 a \text {. }
$$

